\title{
THE UNITED STATES \\ AND THE TRANSFORMATION \\ OF AFRICAN SECURITY: \\ The African Crisis Response \\ Initiative and Beyond
}

\author{
Dan Henk \\ and \\ Steven Metz
}

December 5, 1997 